\title{
A New Super Wideband Fractal Monopole-Dielectric Resonator Antenna.
}

\begin{abstract}
The small physical size and multiband capability are significant in the design of ultrawideband (UWB) antennas. Fractal geometry provides a good method for achieving the desired miniaturization and multiband performances. Furthermore, using a dielectric resonator improves bandwidth and radiation characteristics. A combination of these methods in the UWB antenna design is presented. The proposed design is a new hybrid dielectric resonator antenna (DRA) excited by a new fractal monopole antenna. The simulation and optimization have been carried out using Ansoft HFSS. The simulation and measurement results show that the proposed structure provides a huge bandwidth ranging from 2 to 40 GHz. Radiation patterns and gains show a good agreement over the bandwidth.
\end{abstract}

Keyword: super wideband, monopole, antenna 